\title{
INFLUENCIA DE LOS APARATOS DENTALES ORTODÓNTICOS EN LA PRODUCCIÓN Y PERCEPCIÓN DEL HABLA: ESTUDIO DE CINCO CASOS
}

\author{
The influence of orthodontic dental apparatus in production \\ and perception of speech: study of five cases
}

\author{
Hernán Martínez ${ }^{(1)}$, Elsa Mora ${ }^{(2)}$, Rafael Prato ${ }^{(3)}$
}

\begin{abstract}
RESUMEN
Objetivo: observar la capacidad de cinco individuos venezolanos que usan aparatos dentales ortodónticos para articular el habla y verificar la influencia de los aparatos en la percepción del habla por parte de sus interlocutores. Métodos: se utilizó un corpus conformado por dos grabaciones de la lectura de un Test de Pares Mínimos realizadas por cinco hablantes antes y después de iniciar el tratamiento con los aparatos. Dichas grabaciones fueron luego expuestas a veinte oyentes adultos con la finalidad de que identificaran lo oído; sirviéndose de un test ad hoc, basado en el de los Pares Mínimos arriba indicado. Cada uno de los oyentes marcó la opción que creyó haber percibido. Resultados: el análisis de los datos arrojados por los dos conjuntos de tests permitió determinar los sonidos, los rasgos distintivos y los puntos articulatorios afectados por el uso de aparatos en los hablantes. Se demuestra que los sonidos mayormente afectados por los aparatos dentales son /t/, /m/, /p/, /f/, /b/, / / /, / / / y / t f/; los rasgos y oposiciones distintivas afectadas son [grave]: /b - d/, /f $-\mathrm{s} /, / \mathrm{m}-\mathrm{n} /, / \mathrm{m}-\mathrm{n} / \mathrm{y} / \mathrm{p}-\mathrm{t} /$; [sonoro]: /g - k/,/p-b/y/t - d/ e [interrupto]: /t $\mathrm{s}-\mathrm{s} /$; por su parte, los lugares de articulación afectados son bilabial, alveolar, palatal, dental e interdental. Conclusiones: la presencia de los aparatos dentales ortodónticos en la cavidad bucal causa serios problemas en la articulación de los sonidos lingüísticos afectando de esa manera la percepción del habla por parte de los interlocutores. Los problemas de articulación con el paso del tiempo van decreciendo.
\end{abstract}

PALABRAS CLAVE: Aparatos Ortodónticos; Habla; Percepción

\section{INTRODUCCIÓN}

Los dientes son de gran importancia en la vida del hombre, de hecho la pérdida o la mal posición de los dientes es incompatible con ciertos procesos fisiológicos como los: masticatorios, los fonatorios y los de deglución. En el hombre adulto los dientes son 32 , de los cuales 16 corresponden a cada arcada con cuatro incisivos, dos caninos, cuatro premolares y seis molares en cada arcada.

Las anomalías de posición de los dientes, la pérdida dentaria y las alteraciones de la oclusión se

(1) Magíster Scientiae en Lingüística; Universidad de Los Andes Facultad de Humanidades y Educación - Departamento de Lingüística; Doctorado en Lingüística.

(2) Doctora en Lingüística; Universidad de Los Andes - Facultad de Humanidades y Educación - Departamento de Lingüística.

(3) Magíster en Ortodoncia y Ortopedia Máxilofacial; Universidad de Los Andes - Facultad de Odontología; Cátedra de Ortodoncia. han observado desde la época del Hombre de Neardenthal. Sin embargo, de acuerdo con investigaciones históricas y antropológicas, las primeras referencias de dichas anomalías se encuentran en la época de Hipócrates.

Durante la Edad Media se registraron los primeros métodos odontológicos para corregir las anomalías dentarias. En el siglo XVI aparecen al respecto, entre otros, los trabajos de Hemmard, Paré y Purrman.

En Francia, entre 1728 y 1800 , surge la Ortodoncia. Desde sus orígenes el objeto de esta rama de la odontología es el estudio, prevención y corrección de anomalías de posición de los dientes y alteraciones de la oclusión de los maxilares, de problemas de mordida, así como también de guiar la erupción de los dientes y, por ende, de la armonía dentomaxilofacial, con el fin de restablecer la oclusión y las funciones básicas normales que conducirán al equilibrio de las proporciones esqueléticas y dentarias, a la masticación y deglución eficientes, a la respiración, a la fonación y a la estética facial. 
A partir de 1800 el panorama para la ortodoncia cambia fundamentalmente pues aparece el hombre que sin lugar a dudas marca un nuevo rumbo en el desarrollo y evolución científica de esta rama de la odontología: Edward Hartley Angle. En 1897 publica su primer libro Malocclusion of the teeth en el cual establece una clasificación de las anomalías dentales y de oclusión de simple y fácil aplicación superando las ya existentes. Por otra parte, impuso una aparatología propia conocida y utilizada en todo el mundo: primero, el aparato de perno y tubo, luego el arco-cinta y, posteriormente, el arco de canto con bandas y brackets individuales.

Angle, también Case y Kingsley, entre otros, pronto comprendieron que para realizar movimientos dentarios adecuados era necesario idear alguna forma de controlar los dientes individualmente, sin tener que extraer ninguna pieza ni mover toda la arcada dentaria. Esto los condujo al perfeccionamiento de los aparatos removibles y a la creación de aditamentos soldados sobre coronas modificadas o bandas metálicas fijas. Los primeros aditamentos eran sólo espoleares que servían para sostener un alambre. El típico aparato fijo estaba formado por dos bandas "ancla" colocadas en los molares o coronas modificadas, con tubos largos o cubiertas colocadas sobre la superficie vestibular, paralelas al plano oclusal y un arco labial de alambre pesado que seguía al contorno de la arcada dentaria superior o inferior ${ }^{1}$.

Después de Angle, han sido muchos los autores e investigadores que han impulsado el perfeccionamiento técnico y la orientación de la ortodoncia. En la primera década del siglo XX, Pierre Robin desarrolló un aparato para propulsar la mandíbula. A partir de la década del 20, Andresen creó un aparato que permanecía suelto en la cavidad oral, el cual fue denominado activador y su objetivo era lograr el reposicionamiento muscular, óseo y estructural transfiriendo los estímulos musculares a los maxilares y tejidos de soporte. Por otra parte, en la década de los años 30, surge la ortopedia funcional, cuyo propósito era mejorar la oclusión dentaria a través de movimientos que permitían modificaciones musculares y el crecimiento óseo de la mandíbula sin realizar extracciones dentarias y dando prioridad a las funciones del sistema estomatognático. Así mismo, durante esta época aparecieron varios tipos de aparatos ortopédicos (y sus respectivas modificaciones posteriores); entre otros, las placas bimaxilares de Schwarz, el bionator de Balters, el modelador de Bimler, el regulador de funçión de Fränkel y el activador elástico abierto de Klammt ${ }^{2}$.

\section{Efectos de los defectos maxilodentales en el habla}

Además de actuar como cavidad de resonancia, conjuntamente con las fosas nasales y la laringe, la cavidad bucal es el lugar en el que se articulan los sonidos del habla. Algunas veces, dicha cavidad puede presentar anomalías maxilodentarias que conllevan, generalmente, trastornos en la producción del habla. Perelló ${ }^{3}$ plantea que las deformidades dentales influyen en la correcta articulación de los fonemas ya que éstos actúan como válvulas que detienen, enlentecen o liberan el aire fonador durante el proceso de producción del habla. Varios autores han realizado estudios de la etiología de las maloclusiones y la atribuyen a movimientos de deglución y del habla defectuosos. Algunas de las anomalías maxilodentarias más comunes son: mordida abierta, mandíbula retruida en clase II, prognatismo o proyección de la mandíbula, protrusión de los incisivos superiores y sobremordida horizontal o vertical, ausencia parcial o total de los molares superiores permanentes, hendidura labiopalatina ${ }^{4}$.

Todas estas alteraciones maxilodentarias, de manera general, conllevan alteraciones o trastornos de los patrones de pronunciación o de producción de los sonidos del habla. Este tipo de trastornos del habla se caracterizan por la adición o inserción de sonidos que no deberían estar presentes en la palabra; por distorsión o imprecisión articulatoria (lo que dificulta su identificación al alejarse del patrón); por omisión o ausencia de fonemas que forman parte de la palabra; y por sustituciones de un sonido del habla por otro ${ }^{5}$.

El término que engloba este tipo de alteraciones, según los especialistas, es el de dislalia (etimológicamente, significa dificultad en el habla, del griego dis, dificultad y lalein, hablar). La dislalia constituye un trastorno del habla debido a problemas de articulación que tienen lugar por la alteración anatómica de los órganos periféricos de la ejecución articulatoria u orofonatorios (labios, lengua, paladar, velo del paladar, dientes, maxilares) en el cual no existe una entidad neurológica como base. Las dislalias pueden ser congénitas (como la hendidura labiopalatina) 0 adquiridas (como las producidas por traumatismos). Las dislalias comprometen, entre otras cosas, la comunicación de la persona, pues alteran la inteligibilidad de su habla. Si las dislalias son múltiples, a la persona se le entiende poco lo que habla. Esto trae como consecuencia que el individuo dislálico sea objeto de burlas por los que lo rodean, lo que provoca en él alteraciones emocionales y de conducta, haciéndose tímido, introvertido (no querrá hablar para que los demás no se den cuenta de que habla mal), irritable e incluso se crea en él un complejo de inferioridad, todo lo cual limita sus posibilidades en la vida ${ }^{6}$ ).

De manera general, el término dislalia engloba cinco tipos: dislalias linguales, dislalias labiales, dislalias palatales, dislalias maxilares o mandibulares y las dislalias dentales. Las alteraciones maxilodentarias 
que provocan trastornos del habla entran pues dentro de las dislalias maxilares y dentales.

\section{Algunos antecedentes}

En la misma época en la que surgieron los aparatos dentales ortodónticos (mecanismos, fijos o removibles, que generando una fuerza pueden, por su aplicación, desplazar las piezas dentarias o todo un maxilar en maloclusión ${ }^{7}$ ), se iniciaron los trabajos fonoaudiológicos que estudiaban la influencia de aquellos en la producción del habla, pues, como se sabe, la articulación del habla está relacionada con la configuración anatómica de la cavidad oral: si se usa algún tipo de aparato de ortodoncia para corregir alguna alteración maxilodentaria seguramente habrá interferencia en la articulación normal, pues ocurre una alteración de las dimensiones de la cavidad oral. Existe entonces una gran relación entre las anomalías dentomaxilofaciales, el consecuente uso de los aparatos dentales ortodónticos y los trastornos del habla. A partir de la década de los 70 del siglo pasado, los estudios en esta área despertaron un gran interés.

Varios estudios han enfocado los efectos de la cirugía ortognática ${ }^{8-9}$, el tamaño de los arcos alveolares, problemas de deglución y anomalías oclusales en el habla ${ }^{10}$. Numerosos estudios han demostrado la estrecha relación entre las maloclusiones dentarias y las dislalias, considerando que esta patología constituye el segundo grupo de factores causales de los trastornos en el lenguaje. Otros tantos se han detenido en determinar los efectos de los aparatos dentales ortodónticos en la articulación del habla.

Uno de ellos estuvo a cargo de Cleavonne Strutton y Glenn Burkland ${ }^{11}$, titulado "The effect of maxillary retainers on the clarity of speech". En él se estudia la claridad del habla en personas con distintos tipos de aparatos removibles superiores elaborados con material acrílico: unos cubrían completamente el paladar duro mientras que otros no. Los individuos leyeron un test fonéticamente balanceado llamado "Rainbow Passage", el cual fue escuchado por un panel compuesto por ortodoncistas, audiólogos y especialistas en patologías del habla. Los resultados señalan que los retenedores que no cubren completamente el paladar duro favorecen una normal y clara articulación del habla; mientras que los que, por el contrario, cubren el paladar duro dificultan la claridad del habla. Este hecho sugiere que al reducir el tamaño y grosor de las coberturas acrílicas las limitaciones articulatorias serán menores, aumentando, por tanto, la claridad del habla, la comodidad y reduciendo la sensación de gangueo.

Por su parte, en 1996, el grupo de ortodoncistas encabezado por los doctores Bülent Haydar y Gülsün Karabulut ${ }^{12}$ evaluaron, a través de un test de articulación (basado en consonantes y vocales en palabras monosílabas), la habilidad articulatoria de 15 pacientes en el final del tratamiento ortodóntico y al inicio de la utilización de aparatos removibles y retenedores mandibulares y maxilares. El test lo aplicaron en tres fases: 1) el primer día sin los aparatos; 2) 24 horas después y; 3 ) una semana después de la aplicación de los aparatos removibles. Haydar, Karabulut et al. ${ }^{12}$ encontraron significativos desordenes articulatorios en los fonemas / t / y / d / en el primer día con los retenedores mandibulares y maxilares, también notaron desórdenes importantes en el fonema / $\mathrm{k} /$. Sin embargo, los investigadores sugieren que al séptimo día con los retenedores los problemas desaparecen completamente o decrecen a un nivel que no impide la claridad del habla.

En el mismo sentido, el grupo de investigadores encabezado por Carvalho Rodrigues ${ }^{13}$, a partir de la evaluación fonoaudiológica de veinte pacientes adultos, constató que el $80 \%$ de los individuos que usan la placa palatina acrílica presentan alteraciones en el habla. Según los investigadores "a presença da placa palatina é um fator modificador da fala e dos sons que exijam a junção da língua com o palato". Agregan además que "a presença da placa palatina modifica a mobilidade da língua quando em movimentos curtos, cíclicos e sonoros - estalo e vibração".

Este mismo hecho lo comenta Hagiwara ${ }^{14}$ cuando afirma que el uso de la placa palatina acrílica puede ser suficiente para provocar cambios en la postura, en el tono y en los movimientos de los músculos intrínsecos de la lengua.

Stewart y colaboradores ${ }^{15}$, por otra parte, han realizado una serie de estudios en los cuales demuestran la relación entre la aparatología ortodóntica, ya sea fija o removible, y la aparición de las dislalias, observando que con la aparatología removible se hacen más difícil la fonación y la deglución.

En un trabajo que analiza la relación existente entre el uso de aparatología ortodóncica en pacientes con maloclusiones dentarias y las dislalias, Mora \& López Fernánde ${ }^{16}$ encontraron que la aparición de dislalias está relacionada con el uso de aparatología ortodóntica; sin embargo, señalan que éstas disminuyen debido a la intervención del terapista del lenguaje en el tratamiento (lo que demuestra la importancia del tratamiento conjunto ortodoncista-logopeda para la rehabilitación de los individuos) y a la disminución, por tanto, de las anomalías dentarias. Específicamente, los investigadores encontraron alteraciones en la producción de los fonemas $/ \mathrm{r} / \mathrm{y} / \mathrm{s} /$ por parte de personas con anomalías dentarias de las clases I y II de Angle.

Lo mismo afirman Jiménez Ariosa y colaboradores ${ }^{17}$ al evaluar a 8 pacientes con maloclusión dentaria tratados con el activador abierto elásti- 
co de Klammt. Encuentran dislalias asociadas justamente al uso de ese aparato de ortodoncia las cuales desaparecen, junto con las anomalías maxiloentarias, en el $65 \%$ de los casos transcurrido un año de tratamiento ortodóntico.

Miyawaki, Yasuhara \& $\mathrm{Koh}^{18}$, basándose en la evaluación de 111 pacientes adultos, examinaron los efectos que causan los aparatos ortodónticos en la producción del habla y en la deglución. El $76 \%$ de los pacientes considera que estos aparatos son incómodos para pronunciar los fonemas / s / y / t / y para realizar las funciones de masticación y deglución, aunque esta incomodidad disminuye gradualmente con el tiempo. Datos como estos también han sido recogidos por Hunski ${ }^{19}$ y por Lew ${ }^{20}$.

Recientemente, a partir del análisis de imágenes de habla y de deglución recogidas en video, Matsuoka, Santos \& Marchesan ${ }^{2}$ han podido caracterizar los patrones del habla y de deglución en personas con y sin el uso de aparatos dentales ortodónticos. Estadísticamente, "os itens língua baixa, articulação trancada, sobrearticulação, imprecisão articulatória, maior interposição de língua e distorção na emissão dos sibilantes, todos relacionados com a fala, sofreram influência quando do uso dos aparelhos".

Después de revisar los trabajos anteriormente presentados surge el interés particular por observar la capacidad de los individuos que usan aparatos dentales ortodónticos para articular el habla, pues éstos -como se vio- causan problemas en la articulación, influyendo así, en la percepción lingüística debido a un desplazamiento entre el sonido lingüístico producido y el percibido por el oyente.

Desde esta perspectiva los objetivos principales de esta investigación fueron determinar, a través de análisis perceptivos y del análisis de las características articulatorias, cuáles son los fonemas afectados por los aparatos ortodónticos (estableciendo de esa manera los puntos articulatorios en los que la interferencia es mayor o menor), y determinar cuáles son los aparatos dentales cuya interferencia en la producción de los sonidos lingüísticos es mayor o menor.

\section{METODOS}

Se elaboró un Test de Pares Mínimos (T.P.M.). Dicho test está conformado por una lista de pares mínimos (par de palabras cuya diferencia semántica viene dada por un único segmento fonemático distinto, tipo "peso/beso") para el español venezolano. Esta lista fue elaborada tomando como criterio diferenciador el de los rasgos distintivos propuestos por Jakobson y Halle ${ }^{21}$. Estos rasgos distintivos, de base acústica, son los que aseguran la función distintiva a nivel fonológico en el sistema lingüístico.

Posteriormente, se realizó una primera grabación con el T.P.M. a cinco personas antes de someterse a un tratamiento ortodóntico y una segunda del mismo tipo usando aparatos dentales removibles y/o fijos, realizada un mes después del inicio del tratamiento. Las grabaciones fueron tomadas utilizando un grabador Sony tipo periodista WGP-TCM323. Cada grabación tiene una duración aproximada de 2 minutos. Las grabaciones se realizaron en el consultorio de Ortodoncia del Departamento de Odontología del Centro de Atención Médica Integral e la Universidad de Los Andes (CAMIULA).

Para el análisis perceptivo se utilizaron las grabaciones tomadas a los pacientes. Éstas fueron expuestas a veinte oyentes adultos y del mismo origen nacional que los hablantes, con la finalidad de que identificaran lo oído; sirviéndose de un test ad hoc, el Test de Rasgos Distintivos (T.R.D.) basado, por supuesto, en el de los Pares Mínimos arriba indicado, cada uno de los oyentes marcó la opción que creyó haber percibido. Es preciso señalar que el T.R.D. es una prueba experimental de percepción que, mediante una estricta selección del material, proporciona una estimación cuantitativa sobre la capacidad de discriminación perceptiva de un sujeto, así como una información cuantitativa referente a fonemas, rasgos distintivos y bandas de frecuencia afectados. La finalidad de este test es, pues, la de controlar al máximo los factores de respuesta-estímulo al nivel de la palabra hasta reducirlos a una única variable: el elemento más pequeño constituyente del fonema, el rasgo distintivo. EI T.R.D. se elaboró con la misma lista de pares mínimos del T.P.M. Con el análisis perceptivo se busca evaluar la discriminación semántica por parte de los oyentes, lo que daría una medida de la capacidad del oyente para diferenciar perceptivamente los sonidos lingüísticos, además de mostrarnos cuáles son, a nivel perceptivo, los fonemas más afectados por la presencia de aparatos dentales ortodónticos.

Los resultados arrojados por el estudio perceptivo con los oyentes sirvieron para determinar las características articulatorias de los fonemas en el habla de los usuarios con aparatos dentales de ortodoncia. La finalidad de este análisis es justamente determinar cuáles son las zonas en las que los fonemas son articulados por cada hablante.

De entre tantos tipos de aparatos que utiliza la ortodoncia correctiva o funcional, nuestro estudio analiza hablantes sólo con aparatos fijos (básicamente constituidos por un bracket para arquear el alambre -que rodea la arcada dental-y transmitir la fuerza de ajuste a los pacientes en forma adecuada, precisa y eficaz. Así mismo, se usan ban- 
das metálicas en los molares, utilizándose sólo brackets en los incisivos, caninos y aun en los premolares, adheridos a los dientes empleando resinas epóxicas) y/o removibles (la mayor parte de los aparatos removibles constituyen modificaciones de la placa de Coffin y generalmente el principio que rige su utilización es similar al de los aparatos fijos en un momento dado. Estos aparatos, con algunas excepciones, son toscos, dificultan la masticación, la fonación y la deglución.).

Las anomalías dentomaxilofaciales y el tipo de aparato que están usando las personas que conforman el corpus de nuestra investigación se presentan a continuación:

- Hablante 1: presenta apiñamiento ligero en la arcada superior e inferior. Usa aparatología fija correctiva: arco recto, brackets y alambre de acero inoxidable.

- Hablante 2: presenta diastemas en los incisivos superiores. Para la corrección de la anomalía utiliza una placa de material acrílico.

- Hablante 3: presenta clase III con apiñamiento en la arcada inferior, mala relación molar y canina y problemas de empuje lingual. Usa aparatos fijos: brackets, bandas, arco de alambre de acero inoxidable y una rejilla lingual en el maxilar inferior.

- Hablante 4: es un paciente clase II esquelética y dentaria. Para la corrección se está empleando un posicionador para clase II hecho de material acrílico.

- Hablante 5: presenta mordida abierta causada por succión digital y empuje lingual, además de problemas de deglución. Para la corrección dentaria y de empuje lingual utiliza aparatos fijos: brackets, bandas y una rejilla lingual en el maxilar superior.

Todas estas personas dieron su consentimiento para que se realizara el estudio y se publicaran los resultados del mismo. Así lo hizo constar el Comité de Ética del Consejo de Desarrollo Científico, Humanístico y Tecnológico (CDCHT) de la Universidad de Los Andes en la constancia-aval registrado bajo el codigo H-655-01-06-F.

A continuación se expondrán los resultados arrojados por los análisis estadísticos de los datos obtenidos a partir del estudios perceptivo y del estudio de las características articulatorias por paciente y por cada tipo de aparato dental ortodóntico.

\section{RESULTADOS}

En la Tabla 1 se encuentran los mayores y más significativos porcentajes de pérdida de fonemas a nivel perceptivo en cada hablante con aparatos. Hay que decir que sólo se analizaron los fonemas consonánticos en posición inicial de sílaba, pues en nuestra lengua es la posición en la que las oposiciones mínimas y los contrastes informativos correspondientes se perciben mucho mejor.

\section{HABLANTE 1:}

El mecanismo articulatorio defectuoso que provoca la presencia de los aparatos dentales ortodónticos en el hablante 1 afecta, de manera específica y significativa, la percepción de los fonemas bilabiales /p/, /b/ y /m/ por parte de los interlocutores, debido seguramente a la presencia de los brackets que conforman el aparato fijo que esta persona usa. En menor medida se encuentran afectados los puntos articulatorios labiodental, palatal y velar.

Los cambios en los mecanismos articulatorios de los fonemas traen consigo problemas para discriminarlos perceptivamente. Esto sucede porque sus rasgos distintivos se ven afectados por la disposición de los órganos articulatorios pasivos y activos en el proceso de articulación y por la presencia del aparato.

Los rasgos distintivos mayormente afectados por los aparatos en este hablante son: [grave], [sonoro] e [interrupto] (Tabla 2).

El [grave] opone a los pares /f - s/, /m - $\mathrm{n} /$, $/ \mathrm{m}-\mathrm{n} /, / \mathrm{p}-\mathrm{t} / \mathrm{y} / \mathrm{b}-\mathrm{d} /$, y se caracteriza acústicamente por una concentración de energía en las bajas frecuencias como consecuencia de la disposición de los órganos móviles y fijos en la cavidad supraglótica. El rasgo [interrupto] opone al par / t $\int$ - s/ y se caracteriza acústicamente por un silencio en la zona de frecuencias situada por encima de las vibraciones glotales, seguido y/o precedido por una difusión de energía en una amplia banda de frecuencias. Los pares $/ b-p /, / d-t / y / g-k /$, por su parte, se oponen por el rasgo [sonoro], el cual se caracteriza por la presencia de una excitación periódica de baja frecuencia que se refleja en el espectrograma a lo largo de su línea de base (barra de sonoridad), cuyo correlato articulatorio son las vibraciones de las cuerdas vocales.

\section{HABLANTE 2:}

Los mecanismos articulatorios del hablante 2, por su parte, afectan los fonemas bilabiales /p/, /m/, /b/ debido al diastema incisal superior: la posición de los incisivos hace que los labios no lleguen a unirse por completo para crear la oclusión. Así mismo, el fonema alveolar $/ \mathrm{s} /$, los dentales /t/ y /d/ y los palatales $/ \mathrm{t} \int / \mathrm{y} / \mathrm{n} / \mathrm{se}$ encuentran mal articulados por la presencia de la placa acrílica.

Esos problemas articulatorios traen como consecuencia la afección mayoritaria de los rasgos distintivos [grave] y [sonoro] (Tabla 3).

Los pares $/ \mathrm{m}-\mathrm{n} /, \mathrm{f}-\mathrm{s} / \mathrm{y} / \mathrm{p}-\mathrm{t} /$ dejan de oponerse por el rasgo [grave]. $/ g-k /, / d-t / y / b-p /$, por su parte, dejan de oponerse por el rasgo [sonoro]. 


\section{HABLANTE 3:}

En la producción del habla del hablante 3, por otra parte, encontramos mayormente afectados los fonemas bilabiales /p/, /b/, el nasal bilabial /m/, el dental /t/ y el palatal / t $\int /$. La mala producción viene dada por la poca eficiencia de los movimientos articulatorios de la lengua debida a la presencia de la rejilla lingual y de los brackets, elementos que ocupan mucho espacio dentro de la cavidad bucal. Razón por la cual los pares de fonemas que se oponen por los rasgos [sonoro] y [grave] dejan de hacerlo (Tabla 4).

\section{HABLANTE 4:}

Este hablante presenta, del mismo modo, un déficit articulatorio que viene dado por el posicionador para clase II. Los fonemas mayormente afectados son los bilabiales /p/ y /b/, el nasal bilabial /m/, el nasal palatal / $\mathrm{n} /$, el velar /k/ y el labiodental /f/.

Ese déficit articulatorio afecta la percepción de los pares mínimos opuestos por los rasgos [grave], [sonoro] e [interrupto] (Tabla 5).

\section{HABLANTE 5:}

Al igual que todos los demás hablantes analizados, el número 5 también presenta un déficit articulatorio producto del uso de aparatos dentales ortodónticos. En este caso, los fonemas /m/, /p/, /t/, /d/, /f/, / j/ y / t J / son los afectados. Al igual que en el caso del hablante 3 , los problemas en la articulación son producto del uso de la rejilla lingual. Este hecho afecta la percepción de los rasgos contenidos en la Tabla 6.

Ahora bien, a partir de la observación y del análisis hemos podido determinar la interferencia e influencia que, en la producción de los sonidos lingüísticos, ejercen los aparatos dentales ortodónticos fijos o removibles.

Como se puede ver en la Tabla 7, los aparatos dentales ortodónticos fijos interfieren e influyen de manera contundente en la producción y por tanto en la percepción por parte de los interlocutores de las oposiciones fónicas analizadas. Este tipo de aparato afecta de manera general la producción y percepción de los fonemas en un 29,15\%. Contrariamente, las placas acrílicas lo hacen de manera general en un 23,72\%.

Estos resultados vienen a confirmar el hecho de que las oposiciones mínimas afectadas por la presencia de estos tipos de aparatos de ortodoncia contienen fonemas que, en su mayoría, son articulados en un espacio comprendido entre los incisivos superiores y el alveolo, zonas que esos aparatos invaden.
Tabla 1 - Fonemas mayormente afectados a nivel perceptivo por la presencia de aparatos dentales ortodóticos y por hablante

\begin{tabular}{l|l|l|l|l}
\hline $\begin{array}{c}\text { Hablante } \\
1\end{array}$ & Hablante 2 & Hablante 3 & Hablante 4 & Hablante 5 \\
\hline$/ \mathrm{p} /$ & $/ \mathrm{m} /$ & $/ \mathrm{t} /$ & $/ \mathrm{m} /$ & $/ \mathrm{t} /$ \\
$45,2 \%$ & $43,7 \%$ & $43,7 \%$ & $48,7 \%$ & $52,3 \%$ \\
\hline$/ \mathrm{b} /$ & $/ \mathrm{f} /$ & $/ \mathrm{b} /$ & $/ \mathrm{k} /$ & $/ \mathrm{m} /$ \\
$33,8 \%$ & $37,8 \%$ & $40,0 \%$ & $45,7 \%$ & $51,5 \%$ \\
\hline$/ \mathrm{m} /$ & $/ \mathrm{p} /$ & $/ \mathrm{p} /$ & $/ \mathrm{p} /$ & $/ \mathrm{f} /$ \\
$32,2 \%$ & $31,5 \%$ & $37,5 \%$ & $42,5 \%$ & $49,7 \%$ \\
\hline$/ \mathrm{fl}$ & $/ \mathrm{s} /$ & $/ \mathrm{f} /$ & $/ \mathrm{f} /$ & $/ \mathrm{p} /$ \\
$32,2 \%$ & $30,4 \%$ & $33,7 \%$ & $33,7 \%$ & $42,7 \%$ \\
\hline$/ \mathrm{k} /$ & $/ \mathrm{t} \int /$ & $/ \mathrm{m} /$ & $/ \mathrm{b} /$ & $/ \mathrm{t} \int /$ \\
$31,4 \%$ & $23,2 \%$ & $32,5 \%$ & $33,7 \%$ & $38,3 \%$ \\
\hline$/ \mathrm{t} \int /$ & $/ \mathrm{k} /$ & $/ \mathrm{t} \int /$ & $/ \mathrm{j} /$ & $/ \mathrm{j} /$ \\
$28,2 \%$ & $22,6 \%$ & $25,8 \%$ & $32,5 \%$ & $36,5 \%$ \\
\hline
\end{tabular}

(Los porcentajes representan los errores de percepción del fonema considerado. Así, por ejemplo en el hablante 1, del $100 \%$ de producciones de /p/, 45,2\% no fueron percibidas correctamente).

Tabla 2- Rasgos distintivos mayormente afectados (Hablante 1)

\begin{tabular}{c|c|c}
\hline $\mathrm{Gr}$ ave & Sonoro & Interrupto \\
$35,3 \%$ & $32,9 \%$ & $23,5 \%$ \\
\hline $\mathrm{h}-\mathrm{d} /, / \mathrm{f}-\mathrm{s} /$, & $/ \mathrm{g}-\mathrm{k} /, / \mathrm{p}-\mathrm{b} /$, & \\
$/ \mathrm{m}-\mathrm{s} /, / \mathrm{m}-\mathrm{n} /$, & $/ \mathrm{t}-\mathrm{d} /$ & $/ \mathrm{t}-\mathrm{s} /$ \\
$/ \mathrm{p}-\mathrm{t}$ & & \\
\hline
\end{tabular}

Tabla 3- Rasgos distintivos mayormente afectados (Hablante 2)

\begin{tabular}{c|c|c}
\hline Grave & Sonoro & Interrupto \\
$31,5 \%$ & $21,6 \%$ & $16,7 \%$ \\
\hline$/ \mathrm{b}-\mathrm{d} /, / \mathrm{f}-\mathrm{s} /$, & $/ \mathrm{g}-\mathrm{k} /, / \mathrm{p}-\mathrm{b} /$, & \\
$/ \mathrm{m}-\mathrm{n} /, / \mathrm{m}-\mathrm{n} /$ & $/ \mathrm{t}-\mathrm{d} /$ & $/ \mathrm{t} \int-\mathrm{s} /$ \\
$/ \mathrm{p}-\mathrm{t} /$ & & \\
\hline
\end{tabular}

Tabla 4- Rasgos distintivos mayormente afectados (Hablante 3 )

\begin{tabular}{c|c|c}
\hline $\begin{array}{c}\text { Sonoro } \\
35,0 \%\end{array}$ & $\begin{array}{c}\text { Grave } \\
24,7 \%\end{array}$ & $\begin{array}{l}\text { Interrupto } \\
18,5 \%\end{array}$ \\
\hline$/ \mathrm{g}-\mathrm{k} / \mathrm{/ \textrm {p }}-\mathrm{b} /$, & $/ \mathrm{b}-\mathrm{d} /, / \mathrm{f}-\mathrm{s} /$, & $/ \mathrm{t} \int-\mathrm{s} /$ \\
$/ \mathrm{t}-\mathrm{d} /$ & $/ \mathrm{m}-\mathrm{n} /, / \mathrm{m}-\mathrm{n} /$ & \\
& $/ \mathrm{p}-\mathrm{t} /$ & \\
\hline
\end{tabular}

Tabla 5- Rasgos distintivos mayormente afectados (Hablante 4)

\begin{tabular}{c|c|c}
\hline $\begin{array}{c}\text { Grave } \\
39,4 \%\end{array}$ & $\begin{array}{c}\text { Sonoro } \\
37,5 \%\end{array}$ & $\begin{array}{l}\text { Interrupto } \\
25,0 \%\end{array}$ \\
\hline $\mathrm{lb}-\mathrm{d} /, / \mathrm{f}-\mathrm{s} /$, & $/ \mathrm{g}-\mathrm{k} /, / \mathrm{p}-\mathrm{b} /$, & $/ \mathrm{t} \int-\mathrm{s} /$ \\
$/ \mathrm{m}-\mathrm{n} /, \quad / \mathrm{m}-\mathrm{n} /$ & $/ \mathrm{t}-\mathrm{d} /$ & \\
$/ \mathrm{p}-\mathrm{t} /$ & & \\
\hline
\end{tabular}


Tabla 6- Rasgos distintivos mayormente afectados (Hablante 5)

\begin{tabular}{|c|c|c|}
\hline $\begin{array}{l}\text { Grave } \\
46,2 \%\end{array}$ & $\begin{array}{l}\text { Sonoro } \\
37,8 \%\end{array}$ & $\begin{array}{l}\text { Interrupto } \\
32,3 \%\end{array}$ \\
\hline $\begin{array}{c}/ \mathrm{b}-\mathrm{d} /, / \mathrm{f}-\mathrm{s} /, \\
/ \mathrm{m}-\mathrm{n} /, \quad / \mathrm{m}-\mathrm{n} /, \\
/ \mathrm{p}-\mathrm{t} /\end{array}$ & $\begin{array}{c}/ \mathrm{g}-\mathrm{k} /, / \mathrm{p}-\mathrm{b} / \\
/ \mathrm{t}-\mathrm{d} /\end{array}$ & It $\int-s /$ \\
\hline
\end{tabular}

Tabla 7- Pérdida perceptiva de rasgos distintivos por tipo de aparato dental

\begin{tabular}{l|c|c}
\hline & APARATOS FIJOS & APARATOS REMOVIBLES \\
\hline [grave] & $35,60 \%$ & $35,20 \%$ \\
\hline [sonoro] & $27,08 \%$ & $22,08 \%$ \\
\hline [interrupto] & $24,79 \%$ & $13,90 \%$ \\
\hline
\end{tabular}

\section{DISCUSIÓN}

De acuerdo con los resultados expuestos se puede observar que los rasgos distintivos mayormente afectados por la presencia de los aparatos dentales ortodónticos son el [grave], el [sonoro] y el [interrupto] (en menor medida lo son el [nasal], el [compacto] y el [tenso]). Dentro del grupo de oposiciones fónicas que se oponen por el rasgo [grave] tenemos /f - s/,/m - j/, /m - $\mathrm{n} /, / \mathrm{p}$ - t/y/b - d/; dentro de las opuestas por el rasgo [sonoro] se encuentran / $\mathrm{p}-\mathrm{b} / \mathrm{,} / \mathrm{t}-\mathrm{d} / \mathrm{y} / \mathrm{k}-\mathrm{g} / ; \mathrm{y}$ el $\mathrm{par} / \mathrm{t} \int \mathrm{s} / \mathrm{se}$ opone por el rasgo [interrupto].

De manera general, la pérdida del rasgo [grave] quizás se deba a que en los mecanismos articulatorios de todos los hablantes los fonemas no presentan un resonador amplio justamente por la presencia de los aparatos dentales ortodónticos. Las oposiciones $/ \mathrm{m}-$ $\mathrm{n} / \mathrm{y} / \mathrm{m}$ - n/ son las más afectadas en la percepción del habla de los hablantes. En principio, el fonema / $\mathrm{m} / \mathrm{se}$ articula en los labios; sin embargo, este hablante tiende a articularlo en un punto intermedio entre los labios y el (pre)paladar, zona esta en la que también se encuentran los puntos articulatorios de / $\mathrm{n} / \mathrm{y} / \mathrm{n} /$. Al modificar $/ \mathrm{m} /$ su lugar de articulación deja de ser [+grave] (rasgo mediante el cual se opone a /n/) y, en algunos casos, [+anterior] (rasgo mediante el cual se opone a/j/), rasgos que lo definen y lo hacen distinto. Por su parte, el par $/ p-t /$ deja de oponerse por el rasgo [grave] justamente porque /t/ adelanta su punto articulatorio hacia un punto intermedio entre los labios y los dientes, se vuelve [+anterior] y, por tanto, también [+grave]. Como se puede ver, la calidad de producción de un fonema dependerá de la forma y el tamaño del espacio del resonador, mientras más cerrado sea el espacio menos grave será el sonido. Justamente, los fonemas /f $-\mathrm{s} /$ se oponen por el rasgo [grave]: /f/ [+grave] y/s/ [-grave]. Los hablantes, de manera general, realizan el fonema /f/ como un sonido similar al del fonema /s/ pues, el espacio de la cavidad oral disminuye debido a la presencia del aparato ortodóntico, de ahí que /f/ se vuelva [+grave] y se confunda con el sonido sibilante. Por su parte, el rasgo [sonoro], que articulatoriamente se caracteriza por la vibración de las cuerdas vocales, se ve afectado por la presencia de los aparatos de ortodoncia por el hecho de que éstos, al ocupar mucho espacio en la cavidad oral, promueven por una parte, movimientos linguales deficientes que le dan al habla una mala calidad acústica y una cualidad perceptiva de gangosa y, por la otra, promueven la postura anormal de las cuerdas vocales, lo que hace que éstas vibren afectando la calidad acústica de los sonidos. Por estas razones, los pares $/ \mathrm{b}-\mathrm{p} /, \mathrm{g}-\mathrm{k} /$ $y / d-t /$ dejan de oponerse por completo cuando los $/ \mathrm{p} /, / \mathrm{k} / \mathrm{y} / \mathrm{t} /$ [+sordos] se hacen [+sonoros].

Particularmente, la pérdida perceptiva de los rasgos distintivos [grave] y [sonoro] en la producción fonética de los hablantes 2 y 4 encuentra su justificación en el hecho de que, por diversas razones, las placas acrílicas reducen el espacio y no ofrecen mucha estabilidad en la cavidad bucal, lo que provoca una limitación en los movimientos de los labios y la lengua afectando así la articulación y la percepción de los sonidos bilabiales, labiodentales, dentales, alveolares, palatales y velares. Este hecho ha sido señalado anteriormente por Matsuoka, Santos \& Marchesan ${ }^{2}$ (cuando caracterizan los patrones del habla y de deglución en personas con y sin el uso de aparatos dentales ortodónticos), por Strutton y Burkland ${ }^{11}$ (cuando señalan que los retenedores que no cubren completamente el paladar duro favorecen una normal y clara articulación del habla), por CarvaIho Rodrigues ${ }^{13}$ y por Hagiwara ${ }^{14}$ (cuando afirman que la presencia de la placa acrílica palatina es un factor que perturba el habla). Todo esto nos lleva a sugerir entonces que al reducir el tamaño y grosor de las placas acrílicas las limitaciones articulatorias serán menores. En los hablantes 3 y 5 los rasgos [grave] y [sonoro] se ven alterados por la presencia de la rejilla lingual pues, por un lado, reduce contundentemente el espacio de la cavidad oral y, por el otro, afecta la posición normal de la lengua en la producción de sonidos [anteriores] (como /d - t/), haciendo que esta se dirija hacia la parte posterior de la cavidad bucal (punto articulatorio de $/ \mathrm{g}-\mathrm{k} /$ ). Aunado a este problema, pareciera que las cuerdas vocales vibran al adoptar una postura anormal produciendo de esa manera, a nivel perceptivo, la sensación de un habla gangosa.

El rasgo [interrupto], por otra parte, se caracteriza articulatoriamente por el bloqueo rápido de la fuente sonora (ya sea por la abertura y cierre rápido del aparato vocal, ya sea por las vibraciones que diferencian a las líquidas interruptas de las líquidas continuas). $/ \mathrm{t} \int \cdot \mathrm{s} /$ es uno de los pares cuyos miembros se oponen por el rasgo [interrupto]. De manera general, en el me- 
canismo articulatorio de / / pt $\int$ imos constatar que el momento de oclusión era excesivamente breve, por ende poco perceptible, y el de constricción, cargado de mucha estridencia. Al no ser percibido el momento de oclusión, /t $\mathrm{J} /$ pierde el rasgo [+interrupto] y se vuelve [+continuo] al igual que /s/. Siendo esto así, no habrá manera, entonces, de distinguir perceptivamente el par $/ \mathrm{t} \int-\mathrm{s} /$.

Se ha podido advertir, además, que los puntos de articulación más influenciados por la presencia de los aparatos dentales ortodónticos son el bilabial, el alveolar, el palatal, el dental y el labiodental. Esto se debe a que en los hablantes 3,4 y 5 los puntos articulatorios alveolar y prepalatal están completamente obstruidos por los aparatos dentales que usan. Los hablantes 3 y 5 están usando una rejilla lingual que no permite contactos ápico-alveolares; el hablante 4, debido a que el posicionador para clase II que usa cubre con material acrílico todo el alveolo, no puede realizar una buena articulación de los sonidos alveolares y bilabiales. No sucede así con los hablantes 1 y 2 . El punto articulatorio palatal se encuentra seriamente afectado por los aparatos dentales en la producción de los hablantes 2 y 4, pues este está completamente cubierto por una capa de material acrílico, lo que hace que el dorso de la lengua se desplace o deslice con facilidad sin llegar a crear un estrecho y fijo contacto con el paladar dejando de producir la presión necesaria que caracteriza al sonido palatal. En los hablantes 3 y 5 el punto articulatorio palatal es, en menor medida, también afectado por las rejillas linguales que usan, pues, éstas no dejan que la masa de la lengua se contraiga correctamente para crear el estrechamiento entre ella y el paladar debido al poco espacio que queda libre en la cavidad oral. Por otra parte, en todos los hablantes el punto de articulación bilabial está influenciado por la presencia de los aparatos dentales ortodónticos fijos (pues, como hemos dicho, los brackets están pegados en la cara externa de las piezas dentarias lo que crea un abultamiento de los labios haciendo que éstos se proyecten hacia adelante y hacia arriba y abajo respectivamente y no logren el contacto estrecho necesario entre los labios para mantener la presión de los sonidos que normalmente se articulan en ese punto) y por los removibles (pues, el volumen de estos aparatos, aunado a la anomalía dentaria, hace que los labios no logren un contacto estrecho). El punto articulatorio dental, al igual que el palatal, está muy afectado por los aparatos. Los hablantes 3 y 5 encuentran dificultad, debido a la rejilla lingual que usan, para articular sonidos en ese punto, pues no logran realizar contactos ápicodentales. El hablante 4 presenta el mismo problema pero en menor medida.
El uso de aparatos dentales ortodónticos produce interferencia en la lengua, los dientes, los labios, el paladar y la mandíbula perjudicando de esa manera la producción del lenguaje.

Los aparatos fijos, desde esta perspectiva, son los que influyen mayormente en la producción y percepción del habla debido a que (en la mayoría de los casos) se combinan varios aparatos (pues no todos los de este tipo tienen el mismo efecto) como en los hablantes 3 y 5 . Sin embargo, es importante señalar que hay una disminución del campo articulatorio y una pérdida perceptiva del habla de los individuos que usan las placas removibles de material acrílico por parte de sus interlocutores, como en el caso de los hablantes 2 y 4 , debido al material y a la disposición del aparato dentro de la cavidad bucal.

Al igual que otros autores ${ }^{12,17}$ se sugiere que la prevalencia de dislalias en los hablantes usuarios de aparatos dentales ortodónticos es temporaria. Con el tiempo, es posible encontrar una reducción significativa de las dislalias debido al mejoramiento del aparato orofonatorio y, sobre todo, a la adaptación de los patrones articulatorios (por parte de los hablantes) a las nuevas dimensiones físicas de la cavidad bucal, favoreciendo de esta manera la articulación de los fonemas.

\section{CONCLUSIONES}

A partir de todos los datos obtenidos se ha podido llegar a las siguientes conclusiones:

a. Los fonemas mayormente afectados por la presencia de los aparatos dentales ortodónticos son: $/ \mathrm{t} /, / \mathrm{m} /, / \mathrm{p} /, / \mathrm{f} /, / \mathrm{b} /, / \mathrm{j} /, / \mathrm{k} / \mathrm{y} / \mathrm{t} \mathrm{f} /$.

b. Los rasgos distintivos afectados por la presencia de los aparatos dentales ortodónticos son el rasgo [grave] $(/ \mathrm{b}-\mathrm{d} /, / \mathrm{f}-\mathrm{s} /, / \mathrm{m}-\mathrm{n} /, / \mathrm{m}-\mathrm{n} / \mathrm{y} / \mathrm{p}-\mathrm{t} /)$, el [sonoro] $(/ \mathrm{g}-\mathrm{k} /, / \mathrm{p}-\mathrm{b} / \mathrm{y} / \mathrm{t}-\mathrm{d} / \mathrm{)}$ y el [interrupto] $\left(/ \mathrm{t} \int-\mathrm{s} /\right)$.

c. Los puntos articulatorios más influenciados por la presencia de los aparatos dentales ortodónticos son: el bilabial, el alveolar, el palatal, el dental y el interdental.

d. Los aparatos de ortodoncia que mayor interferencia e influencia ejercen en la producción del habla son, en nuestro caso particular de estudio, los aparatos para clase III combinados para corregir problemas de deglución, empuje lingual y succión digital, es decir, los aparatos fijos que utilizan o combinan la rejilla lingual. En menor medida lo hacen los aparatos removibles de material acrílico que cubren toda la zona palatina. Sin embargo, hay que señalar, además, que los aparatos fijos que no combinan la rejilla lingual causan menos problemas articulatorios que los aparatos removibles, 
caso del hablante 1, por ejemplo. El habla se verá perjudicada entonces si el aparato de ortodoncia ocasiona alteraciones en la dimensión vertical de oclusión, si invade el paladar duro y el blando y si no brinda estabilidad en la cavidad oral.

Efectivamente, con los datos obtenidos se demuestra que los aparatos dentales ortodónticos, independientemente de su función, causan serios problemas en la articulación de los sonidos lingüísticos. Sin embargo, hay que decir que los problemas articulatorios en el habla con el paso del tiempo van decreciendo ya que, ante las nuevas dimensiones físicas de la cavidad oral, los hablantes reorganizan los puntos articulatorios para lograr una articulación fonética adecuada.

La terapia logopédica servirá, sin lugar a dudas, para lograr la correcta articulación de los fonemas aun con el uso de los aparatos dentales ortodóticos.

\section{ABSTRACT}

Purpose: to observe the capacity to articulate speech in five Venezuelan individuals who use orthodontic dental apparatus and to verify the influence of the apparatus on the perception of speech from their interlocutors. Methods: the corpus of study consists of two recordings of the lecture of a Minimal Pairs Test done by five speakers before and after beginning the treatment with the apparatus. These recordings were submitted to twenty listeners who were asked to answer what they have perceived. Each one of the listeners checked the option that they believed to have perceived. Results: data analysis showed that sounds, distinctive features and places of articulation are affected by using apparatus by the speakers. The sounds mostly affected by the dental apparatus are $/ \mathrm{t} /, / \mathrm{m} /, / \mathrm{p} /, / \mathrm{f} /, / \mathrm{b} /, \mathrm{j} /, / \mathrm{k} /$ and $/ \mathrm{t} \int /$; the distinctive features affected are [grave]:/ $\mathrm{b}-\mathrm{d} /, / \mathrm{f}-\mathrm{s} /, / \mathrm{m}-\mathrm{n} /, / \mathrm{m}-\mathrm{n} /$ and $/ \mathrm{p}-\mathrm{t} /$; [sonorous]: $/ \mathrm{g}-\mathrm{k} / \mathrm{/} / \mathrm{p}-\mathrm{b} / \mathrm{and} / \mathrm{t}-\mathrm{d} / \mathrm{and}$ [interrupted]:/t $\mathrm{s}-\mathrm{s} /$; the places of articulation affected are bilabial, alveolar, palatal, dental and interdental. Conclusions: the existence of orthodontic dental apparatuses in the oral cavity causes serious problems in the articulation of the linguistic sounds affecting in that way the perception of speech from the interlocutors. Articulation problems decrease with time.

KEYWORDS: Orthodontic Appliances; Speech; Perception

\section{REFERENCIAS}

1. Graber TM. Ortodoncia, teoría y práctica. México: Interamericana; $1974.893 \mathrm{p}$.

2. Matsuoka E, Santos DAG, Marchesan IQ. Padrões de fala e de deglutição em usuários de aparelho ortopédico funcional com e sem o uso do aparelho. Rev CEFAC. 2006; 8(2):198-204.

3. Perelló J. Trastornos del habla. Barcelona: Editorial Científico-Médica; 1981.528 p.

4. Martínez H. La articulación del habla en individuos con hendiduras labiopalatinas corregidas: estudio de dos casos. Rev CEFAC. 2006; 8(2):186-97.

5. Marchesan IQ. Fundamentos de fonoaudiología: aspectos clínicos de la motricidad oral. Buenos Aires: Editorial Médica Panamericana; 2002. 127 p.

6 . Bermúdez D. El lenguaje y su patología. Maracaibo: Serie del Centro Médico de Investigaciones de la Audición, la Voz y el Lenguaje; 1998. 209 p.

7. Guardo A, Guardo C. Ortodoncia. Buenos Aires: Editorial Mundi; $1981.1984 \mathrm{p}$.

8. Dalston RM, Vig PS. Effects of orthognathic surgery on speech: a prospective study. Am J Orthod. 1984; 86(4):291-8.

9. Bowers J, Tobey EA, Shaye R. An acoustic-speech study of patients who received orthognathic surgery.
Am J Orthod. 1985; 88(5):373-9.

10. Garber SR, Speidel TM, Marse G. The effects on speech of surgical premaxillary osteotomy. Am J Orthod. 1981; 79(1):54-62.

11. Stratton CS, Burkland GA. The effect of maxillary retainers on the clarity of speech. J Clin Orthod. 1993; 27(6):338-340.

12. Haydar B, Karabulut G, Özkan S, Aksoy AU, Ciger

S. Effects of retainers on the articulation of speech. Am J Orthod Dentofacial Orthop. 1996. 110(5):535-40. 13. Rodrigues AC, Turra GS, Pires MH, Senandes NC, Chiappetta AL. A influência do uso de cobertura palatina acrílica na cavidade oral. Rev CEFAC. 2004; 6(1):29-33.

14. Hagiwara M. Relation between articulatory function and prosthesis - II-1 Longitudinal changing aspect of experimental palatal plate on muscular discharge of intrinsic muscles, tongue movements and mandibular movement before pronunciation. Nihon Hotetsu Shika Gakkai Zasshi. 1990; 34(3):467-79.

15. Stewart FN, Kerr WJ, Taylor PJ. Appliance wear: the patient's point of view. Eur J Orthod. 1997. 19(4):377-82.

16. Mora C, López Fernández R. Aparatología ortodóncica y trastornos del lenguaje. Rev Cub Ortod. 2001; 1:38-46. 
17. Jiménez Ariosa A, Acosta Basnueva B, Soto Cantero L, Fernández Collazo L. Alteraciones del habla en niños con anomalías dentomaxilofaciales. Rev Cub Ortod. 1997; 13(1):29-36.

18. Miyawaki S, Yasuhara M, Koh Y. Discomfort caused by bonded lingual orthodontic appliances in adult patients as examined by retrospective questionnaire. Am J Orthod Dentofacial Orthop. 1999; 115(1):83-8.
19. Hunski M. Speech disturbance (dyslalia) and orthodontic abnormalities. Acta Stomatol Croat. 1988; 22(4):251-9.

20. Lew KK. Attitudes and perceptions of adults towards orthodontic treatment in an Asian community. Community Dent Oral Epidemiol. 1993; 21(1):31-5. 21. Jakobson R, Halle M. Fundamentos del lenguaje. Madrid: Ayuso; 1973. 245 p.

RECEBIDO EM: 12/09/06

ACEITO EM: 02/11/06

Endereço de correspondencia:

Universidad de Los Andes

Facultad de Humanidades y Educación

Centro de Investigación y Atención Lingüística (CIAL)

Av. Las Américas, Conjunto La Liria, Edificio D, 2do piso

Mérida 5101, Venezuela

Tel: +582742401784

Fax: +582742401784

E-mail:hjmart@cantv.net 